\title{
Image-type representation: A preliminary study on preferences of users with intellectual disabilities
}

\author{
Tânia Rochaa ${ }^{\mathrm{a}, *}$, Maximino Bessa ${ }^{\mathrm{a}}$, Rute Bastardo ${ }^{\mathrm{b}}$, Luís Magalhães ${ }^{\mathrm{a}}$ \\ a University de Trás-os-Montes e Alto Douro and INESC TEC Portugsl \\ ${ }^{\mathrm{b}}$ University de Trás-os-Montes e Alto Douro Portugsl
}

\section{A R T I C L E I N F O}

\section{Keywords:}

User-centered design

Image-type representation

People with intellectual disabilities

Digital content

\begin{abstract}
A B S T R A C T
Previous findings have shown that users with intellectual disabilities navigate the Web more easily and with greater interest when images are used to represent hyperlinks (Rocha, 2008; 2014; Rocha et al., 2012). Although images can be better for navigation purposes, there is a need to understand how these images should be designed to enhance users' interaction with digital content for this particular group of people. The purpose of this study is to measure the user's preference for image-type representation (Object, Action and Universe), within four different categories (music, movies, sports, games). The sample consisted of 20 individuals with intellectual disabilities, their ages ranging from 22 to 49 years old. The results showed that they preferred less complex images, namely object representative images, and that categories had no effect.
\end{abstract}

(C) 2017 Elsevier Ltd. All rights reserved.

\section{Introduction}

Since 2008, we have collaborated with an institution that develops activities with the main goal of enhancing educational and professional skills of people with intellectual disabilities. This collaboration intended to study the development of assistive technologies and accessible contents and, ultimately, to enhance digital skills of a group of people with intellectual disabilities. In this context, we find that people with intellectual disabilities have great motivation and will to access digital content (Rocha, 2008; Rocha et al., 2009; 2012a). Moreover, we have observed that when users with intellectual disabilities navigate through Web content, they pay more attention to and show more interest in images and neglect textual content (we have observed that they tend to click on several image hyperlinks but scarcely click on text hyperlinks) (Rocha, 2008; Rocha et al., 2012b).

Subsequent studies showed one of the major problems: they cannot autonomously perform a Web search task to find pre-defined digital content through the usual metaphor, i.e., by using the search field of a Web search engine and inserting a keyword (Rocha et al., 2012b; Rocha, 2014; Rocha et al., 2015). Thus, the usual metaphor for Web search (i.e., inserting keywords) is not accessible to users with intellectual disabilities because they have low literacy skills with respect to writing and reading (and for this task, they must identify/recognise keyword characters and respond by using the keyboard) (Harrysson et al., 2004; Roh, 2004; Small et al., 2005; Carey et al., 2005; Bohman, 2007; Rocha, 2008; 2014).
These outcomes motivated us to study the use of images to represent hyperlinks on the web. Images should be easy to identify and understand to ultimately enhance the user interaction with Web content, by circumventing the reading and writing abilities needed for this specific task for this specific group of users.

In the literature, we found that the recommendations for making Web content more accessible, the Web Content Accessibility Guidelines, version 2.0, provided by the World Wide Web Consortium (W3C-WAI 2.0), do not define specific guidelines for designing image hyperlinks; they only suggest that alternative text must be provided for any nontext content (WAI, 2008). Also, we discovered that several authors have examined the use of images to navigate or search content (Uden and Dix, 2000; Dix et al., 2004; Sevilla et al., 2007; Freidman and Bryen, 2007; Zarin, 2009), and others described the image categories tested (Wiebe et al., 2016). However, they did not provide any explanation regarding the image design process (How should images be represented? Which composition they should have?). Thus, it is important to address the design of images to study the best way to represent hyperlinks.

As we focus on a specific group of users, people with intellectual disabilities, we intend to base the study on a user-centred design philosophy. User-centred design, also known as Usability Engineering, is the philosophy of achieving more usable systems (reducing errors, improving productivity, user acceptance, and satisfaction) that involves designing interfaces to fit the capabilities and needs of the users as an alternative to the previous philosophy of technology-centred design that required adaptation by the human (user) (Endsley, 2016).

\footnotetext{
* Corresponding author.

E-mail address: trocha@utad.pt (T. Rocha).
} 
In sum, our main objective is to measure the user's preference for image-type representation (Object, Action, and Universe), within four different categories (music, movies, sports, games) throughout their preferences, by investigating which are their mental models.

Users' preferences as included in the user experience (UX) philosophy focus on having a deep understanding of users, what they need, what they value, their abilities, and also their limitations (usability. gov). Moreover, as described in the ISO 9241-210 definition "user experience includes all the user' emotions, beliefs, preferences, perceptions, physical and psychological responses behaviours and accomplishments that occur before, during and after use" (ISO 9241-201, 2010).

This paper is structured as follows: first, the image-type representation design is explained, where we describe the models followed in the icon design process; then, the method section provides detailed information regarding the participants (sample), materials and apparatus and the procedures; afterwards, the results are presented; followed by their discussion, where we underline the study limitations; and, finally, we present our conclusions and future work.

\subsection{Image-Type representation design}

As Nadin (1988) affirms, "to design means to structure systems of signs in such a way as to make possible the achievement of human goals: communication (as a form of social interaction), engineering (as a form of applied technical rationality), business (as a form of shared efficiency), architecture, art, education, and etcetera." (Nadin, 1988).

This study does not have as its main focus on semiotics, rather, we intend to study users' preference for image-type representation within specific categories for people with intellectual disabilities. It is not intended to determine which specific sign is the best to represent a category, because, although "Design principles are semiotic by nature", we must understand how images are decoded to present solutions for imagetype representation design. For that, we present the definition of sign by Peirce and Saussure.

Charles Peirce (American philosopher) advanced the definition of sign as "something that stands to someone for something in some respect or capacity" and defines the sign as a triadic relation between the sign vehicle (the physical form of the sign), a sign object (the aspect of the world that the sign carries meaning about) and an interpretant (the meaning of the sign as understood by an interpreter).

However, Ferdinand Saussure (Swiss professor of linguistics) has proposed a different definition for what a sign is: the unity and combination of a signifier and the signified (Bouquet, 2004; Saussure, 1996). Saussure has advanced the definition of signs as the unity between a signifier (the actual sign embodied in some material form, such as words or shapes) and the signified (what the sign is supposed to mean), becoming a dyadic relation. Saussure saw this relation as being essentially arbitrary, motivated only by social convention.

Peirce's triadic model presented may serve as an operational model to decode a sign. As this author determines only the unity among the three components, Object, Representation and Interpretation, that represents a sign. Furthermore, according to this author, a sign has also three functions: Semiotics as science of representation, science of expression and science of knowledge. Specifically, Semiotics as science of representation implies the Representation and Object, i.e., representation based on resemblance, likeness, indexically (representation causally influenced by the object); science of expression implies a relation between Representation and Interpretation, i.e., the relation between signs and the user, defining what signs are used for; and, the science of knowledge implies Interpretation and Object (based on acknowledging diversity, conventions, meaning).

The components and functions of a sign are evident in Fig. 1, by the arrows ( $\mathrm{O}$ to $\mathrm{R}$ - semiotics as a science of representation; $\mathrm{R}$ to I semiotics as a science of expression; and I to $\mathrm{O}$, semiotics as a science of knowledge) (Nadin, 1988).

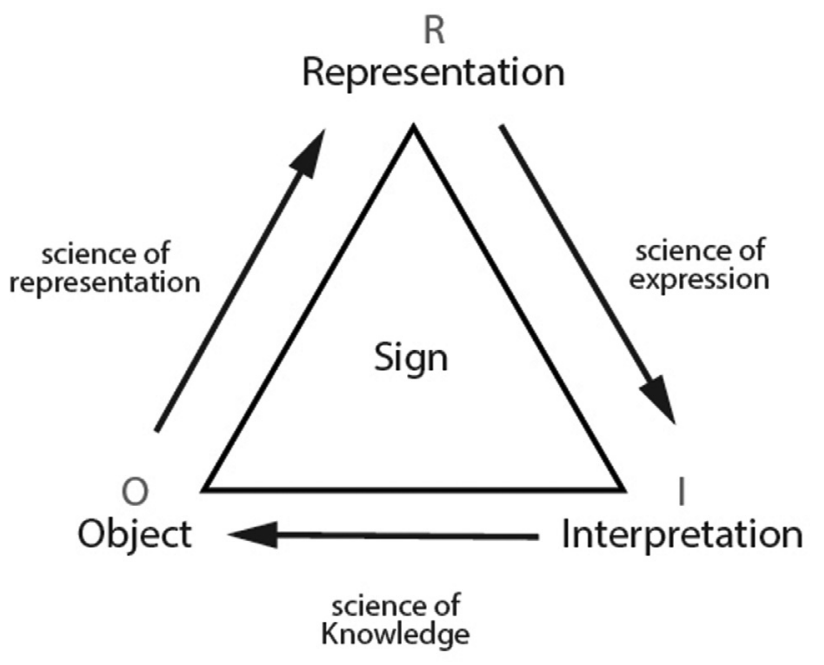

Fig. 1. Peirce's operational model (three components of a sign: $R-$ Representation $=$ that which represents; $\mathrm{O}-$ Object $=$ that which is represented; and $\mathrm{I}-$ Interpretation $=$ the process of interpretation).

Furthermore, the two major theories (Peirce's triadic and Saussure's dyadic models) describe the way signs gain the ability to transmit information. Both theories perceive that the defining property of the signs is a relation between several elements. According to both theories, "signifier" is a representation that is closer to the real or the factual, whereas "signified" is a representation that is farther from the real, closer to the associated concepts. Thus "signified" is harder to decode. In Fig. 2, three different examples of music icon representation are shown. Icon representation 1 , which is closer to the "signifier", is a more real, factual, objective representation, which is simpler to recognise. Icon representation 2 combines its graphical representation with its interpretation (mode of use, action). Icon representation 3, which is closer to the "signified", is more complex in meaning, more symbolic and abstract since it is not tangible. The musical note image is a more complex way to represent music and therefore there is a higher level of decodification associated with it.

Specifically, in Fig. 3, we present the Peirce's operational model, relating the components and functions of the sign with a music-type representation example. This relation led us to consider three imagetype representation formats, which are: Object, Action, and Universe. To better understand these formats, we present the following example: Music icon representation. We can directly associate a headphones sign with two components of the sign, the object and representation (O-R) (which we called OBJECT because of its close connection to the representation). Additionally, we associate a person listening to music with the headphones sign with two sign components, the representation and interpretation (R-I) that impose expression and movement (which we called ACTION). Finally, we associate a musical note sign with the two remaining components, object and interpretation (O-I), which requires knowledge for their decoding (which we called the UNIVERSE).

In short, we sought to evaluate different types of image representations (Object, Action and Universe) to understand whether there is a type of representation that is preferred by people with intellectual disabilities.

\section{Methods}

This is a quasi-experimental, case-control study with a quantitative focus. Its purpose is to investigate the impact of image-type and icon category on the user's preference. 


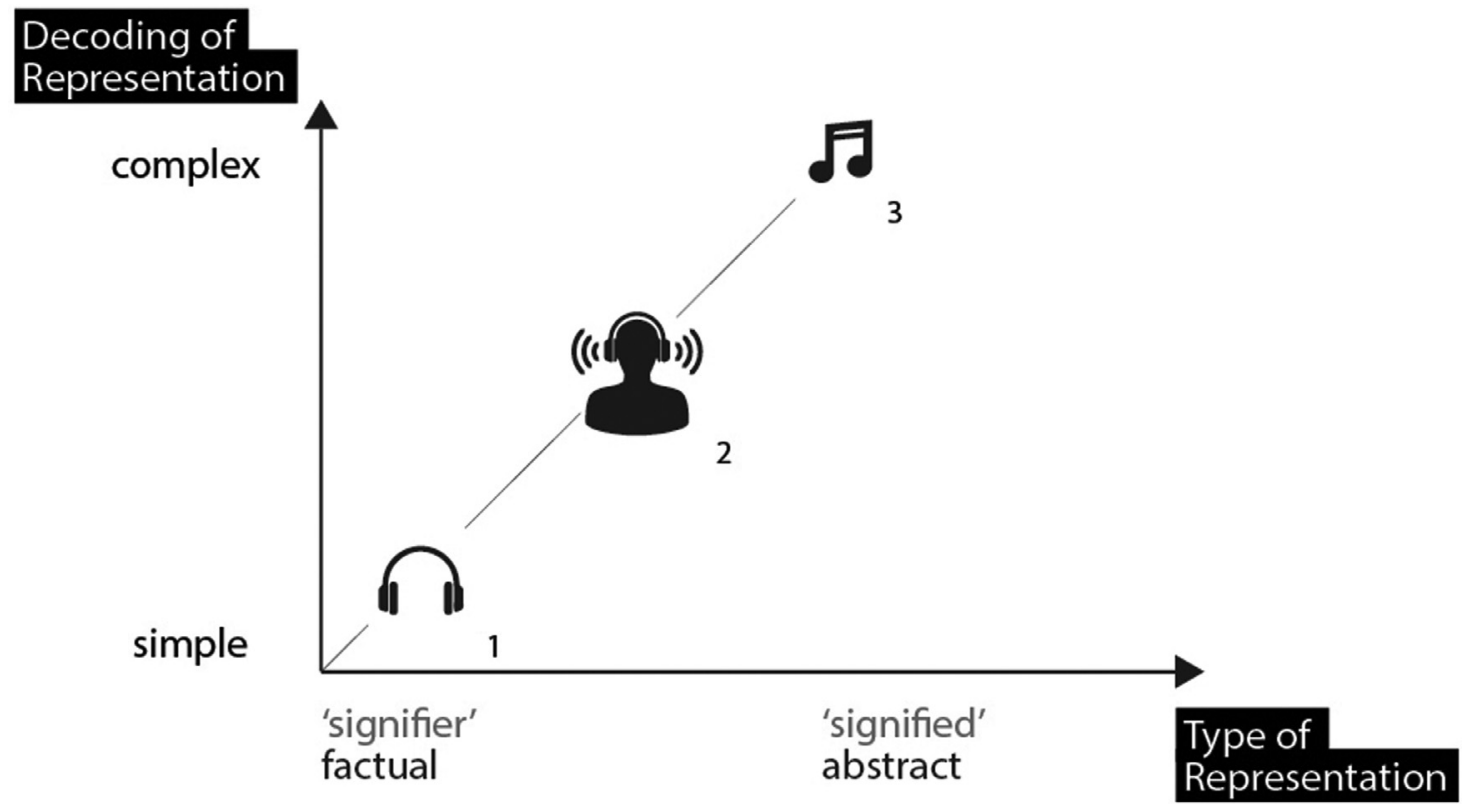

Fig. 2. Type representation vs decoding. example with music icon representation.

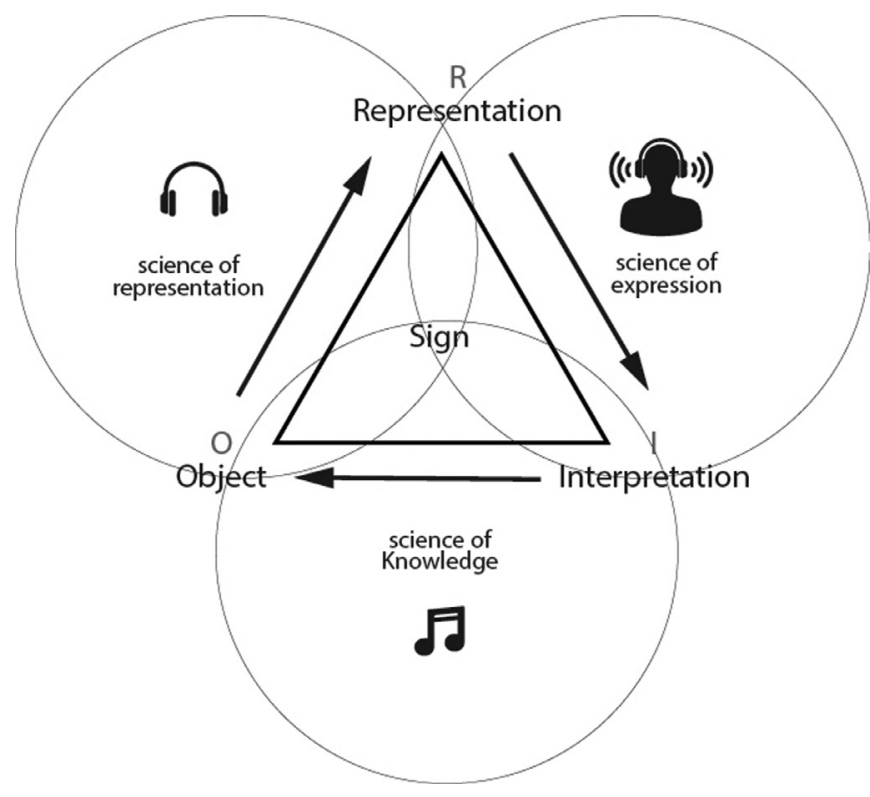

Fig. 3. Operational model - music icon representation example.

\subsection{Sample}

Within the intellectual disabilities it is very difficult to achieve a homogeneous group of participants (even with the same pathology), as they may have several pathologies and levels of severity. Therefore, we identified other characteristics, such as: their literacy level and digital skills (specifically, their level of computer and internet interaction), to select the participants, hoping this way we could get a more cohesive group. Thus, participants were not chosen only on the basis of their type or level of disabilities but were selected to ensure the same level of literacy and techno-abilities within the group.

Specifically, twenty individuals with intellectual disabilities partook in this study, with ages ranging from 22 to 49 years old (eight women and twelve men). The participants were selected by a special education teacher and a psychologist, that worked with them on a daily basis. They divided the participants according to their level of learning through a diagnostic test and knowledge of their reading and writing skills (developed in school context). Their literacy level varied between kindergarten and primary education; specifically, fifteen had a kindergarten-level of literacy, and five had levels between the first and second years of primary school, as is defined in the Portuguese education system that is equivalent to the elementary school in USA and Canada. Within the group, seven users had writing and reading skills correspondent to the first and second year levels and the others could only read and write their names and identify all graphemes (characters) autonomously. Also, they were chosen according to the level of autonomy in the accomplishment of personal daily tasks, such as: hygiene, feed, and other daily personal routines.

Regarding the intellectual disabilities, the psychologist provided global information regarding the participants' pathologies and their severity levels (according to DSM-V 2013). Specifically, eight participants had foetal alcohol syndrome, five were identified as being in mild and three at moderate severity levels; eleven participants had Down Syndrome, one had a severe and ten mild intellectual impairment; one participant had meningococcal disease (known as bacterial meningitis) and developed a moderate cognitive impairment (problems with thinking, learning and making decisions). Additionally, within the group, fourteen participants had normal vision, and six had corrected-to-normal vision.

Concerning the computer and Internet interaction, all participants partook in a digital integration program that had started in their school in 2008. This program allowed them to train and acquire technological abilities, specifically, in the use of basic features with the computer (such as: switch on and off, open the browser, maximise a window, open a specific software), training of Web selection, manipulation and navigation tasks. Also, they used several educational and entertainment platforms. All these activities gave them abilities with the mouse and keyboard handling and are detailed in Rocha's studies (Rocha, 2008; Rocha et al., 2009; Rocha, 2014; Rocha et al., 2015).

All participants were volunteers and had permission (in the form of a written consent) from their parents or tutors to perform the tests. 


\subsection{Materials and apparatus}

The icons selected for this case study were created taking into consideration Uden and Dix (2000) guidelines. These authors stated the importance of creating iconic interface systems, addressing the user mental model and that icons should be designed to look like familiar objects, corresponding to the users' mental models (Uden and Dix, 2000).

In accordance with this statement, we needed to understand which were the users' mental models before we started creation of the icons. In this regard, we conducted individual interviews with the participants and a special education teacher, in which we asked participants to list their favourite activities (e.g., listening to music, going to the swimming pool, drawing and painting, playing football, sewing, watching movies, and doing laundry and kitchen activities).

Afterwards, we invited participants with intellectual disabilities to perform a drawing activity, in which they had to make drawings according to four established categories, music, games, sports, movies, as we intended to unveil which kind of representation they were more familiarised with, revealing data regarding their mental models.

The major results found of this activity were that they drew images according to the three types of representations: "Object"; "Action"; and "Universe". Specifically, in the first representation type, we included all the icons that represent objects; this category relates to the "O to R" side of the triangle in Fig. 3, because the participants knew the object and drew a representation of it. This type of representation also corresponds to the simpler form, as shown in Fig. 2. The "Action" type of representation comprised all the drawings that expressed any form of action. This action was perceived from icons in which simple objects were decorated with strokes that indicated a participant's own representation of the functionality of that object (for example, the participant might indicate that the object "projector" has been decorated with strokes in front of it to represent the light coming out of the projector), or the combination of elements promoting the sensation of action. This type of representation relates to the "R to I" side of the triangle in Fig. 3 and denotes a more complex form of representation, as shown in Fig. 2. Finally, the "Universe" type of representation was defined to be the umbrella for the drawings that implied knowledge of the context of the category (sometimes also known as the "universe" of that category). This category is much more complex and is therefore harder to decode. For example, in the music category, some extended knowledge is needed to represent a sound with a musical note, a sound wave or a histogram. This relates to the "I to O" side of the triangle of Fig. 3 and to the most complex form of representation shown in Fig. 2. This information was important, because it allowed to minimise the number of icons designed according to the suggestions of their drawings.

Moreover, as the participants were familiar with different types of representations through their daily activities (experience in non-digital, digital and Web contexts), we selected images that participants had seen or interacted with directly or in the context of a digital or non-digital interface.

Considering the collected information (interviews, drawing activity, and Portuguese social and cultural digital context), we tailored the design of the icons to fit into one of four categories: music, movies, sports, and games. Fig. 4.

In sum, we used the data provided from the drawing activity and included other relevant icons found in a Web context. For instance, we found several icons for theatre on the web like "an eye on a screen" or "people watching a movie" when searching for theatre icons. These icons are designed with the combination of several elements promoting the act or the action you want to convey.

All images were icons in duotone (black and white), because we intended to assess only image representation and no other characteristics, such as colour, artwork, or realism of the representation (these variables will be considered in future work).

The following material resources were used: a 17 inch display with resolution of $1280 \times 1024$ pixels and an eye tracking device. Each sub-
Table 1

Chi-square test results ( $\mathrm{p}$ value).

\begin{tabular}{lllll}
\hline & $\chi^{2}$ & $d f$ & $\mathrm{P}$ & Cramer's V \\
\hline Music & 12.4 & 2 & 0.002 & 0.557 \\
Movies & 9.7 & 2 & 0.008 & 0.492 \\
Games & 14.8 & 2 & 0.001 & 0.608 \\
Sports & 11.2 & 2 & 0.004 & 0.529 \\
\hline
\end{tabular}

ject sat down at a distance from the computer screen that did not exceed one meter. The individual's eye movements were recorded by a Tobii Eye-Tracker X50 (Tobii Technology AB, Danderyd, Sweden), which used a CCD camera positioned under the computer screen, and the software Clear View 2.5.1.

\subsection{Procedure}

The experimental setup was conducted with one participant at a time. Before starting the tests, the eye tracking device was calibrated. If the calibration step was successful, all images, for each category, were displayed on the screen, without scrolling, as has been suggested in other studies (Roh, 2004; Freeman et al., 2005; Small et al., 2005). Only images were presented on the screen, there were no labels regarding category or image-type. The researcher asked "what image do you prefer" for a particular category.

Then, participants were asked to choose the image that best represented each category by pointing to the screen. This procedure was performed to simplify the process of choosing and to eliminate the mouse interaction complexity.

All participants performed four tasks, each one related to the four categories (music, movies, games, and sports). To ensure randomness, we changed the order in which the categories were shown.

\section{Results}

Fig. 5 shows the overall results of the image-type representation choices. There was a strong preference for Object image-type representation, followed by Action image-type representation, in each category. Additionally, there was little or no preference for images representing the Universe image-type representation, and in two categories of games and sports, there was not a single choice for such images. The variables, icon category and image-type representation, were both categorical, and since each participant made a choice in each icon category, they were considered to be matched groups. Thus, a repeated measures logistic regression test was used to identify whether the icon category had an influence on the selected image-type representation. The results indicated that the icon category was not statistically significant (Wald Chi-Square $=5.943, p=0.114$ ) in influencing the selected image-type representation, as presented in Fig. 5.

The results were analysed using Chi-square goodness of fit tests to determine whether the observed data resulted from a simple random event (meaning that we could not reach a conclusion). Thus, the observed values were tested against the expected values, in which all types of representation had equal probabilities. Table 1 presents the Chi-square test results.

For each icon category, the Chi-square test conditions were met (Yates et al., 1999). In this particular case, all expected counts were greater than 5. In all categories, the null hypothesis was rejected ( $\mathrm{p}<0.05)$; hence, the observed data did not originate from a simple random event.

In Fig. 6, we present the detailed results of the Music category choices. The subjects selected a total of seven different images. The top three images were piano (object), which was selected by eight people; drum (object), selected by three people; and the graphic showing a person listening to music (action), also selected by three people. Globally, 


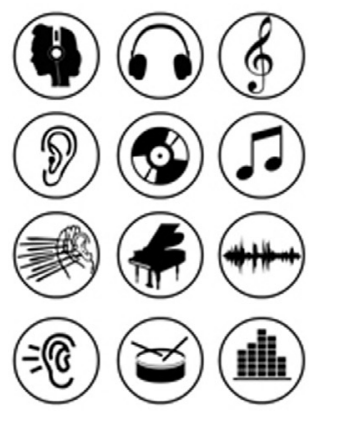

Music Icons

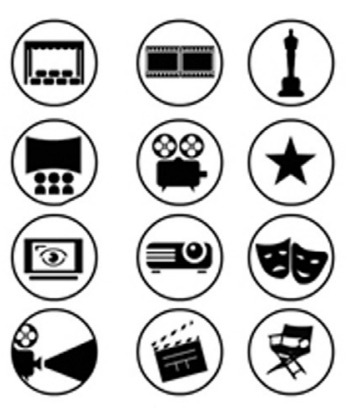

Movies Icons

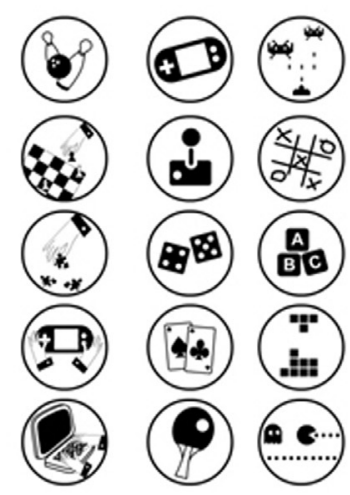

Games Icons

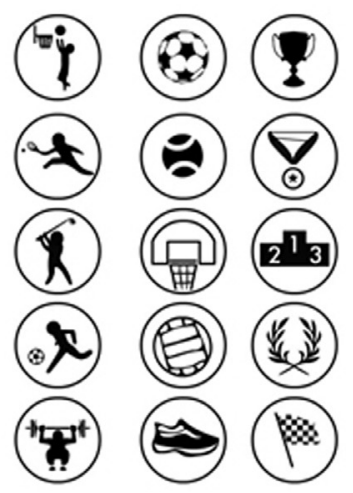

Sports Icons

Fig. 4. Images created for the different categories.

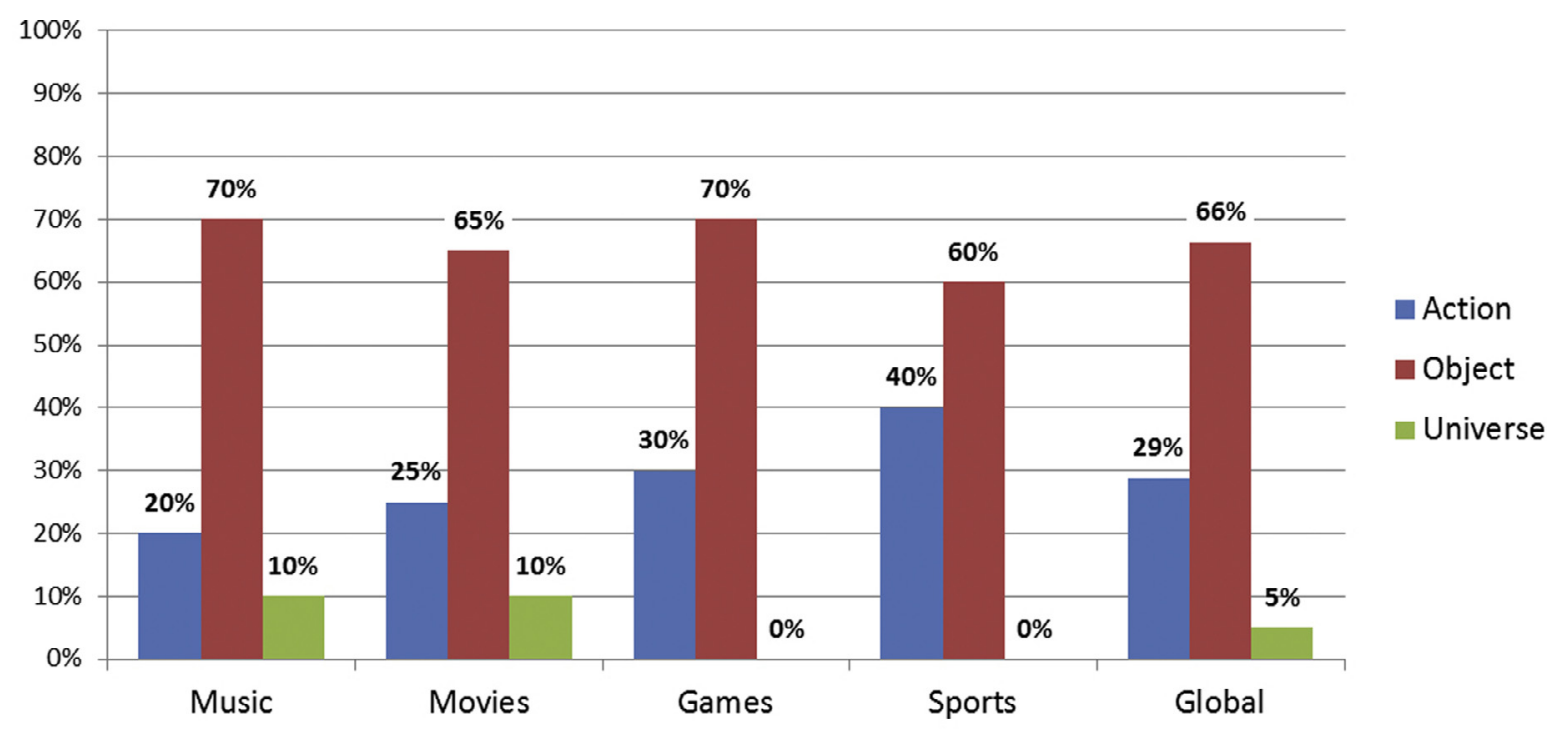

Fig. 5. Global results for choice of image representation.

the participants preferred $70 \%$ icons related to objects; $20 \%$ action; and $10 \%$ universe.

Regarding the movies category, the results are summarized in Fig. 7. Results showed a wider range of choices, i.e., ten images out of a total of twelve images. The most selected images were the video camera (object), which was preferred by eight individuals, followed by the clapperboard (object), film (object), and cinema (action), with two selections each. In the top choices, three referred to the objects, and one referred to the action. Overall, participants preferred $65 \%$ icons related to objects; $10 \%$ action; and $15 \%$ universe.

In Fig. 8, we present the detailed results of the games category. From fifteen icons, seven images were selected by the participants: the two cards icon (object) was the most selected one (seven choices); two dices icon (object), racket and ball (object), and playing chess (action) all had three selections each. As shown in the Fig. 8, globally, the participants preferred $75 \%$ icons related to objects; and $25 \%$ action.

The results for sports category are presented in Fig. 9. From a total of fifteen icons, six images were selected. Participants selected the soccer ball (object), with seven selections; the person playing football image (action), with five selections; the tennis ball (object), with three selections. Among the top choices, two referred to the objects and one referred to the action. Overall, the participants had a $50 \%$ preference regarding object, and $50 \%$ action.
Next, we present the results from the eye tracker data. As mentioned before, the eye tracking device was used as a validation tool to ensure that participants actually chose the image that they paid attention to. The results yielded insights regarding the strategies they used to complete the task. We found that the subjects explored most of the icons, including the universe type of representations. The heat maps (agglomerated analysis of the visual exploration patterns of a group of users) are shown below (Fig. 10).

For each icon an Area of Interest (AOI) was defined. The icons/AOIs were labelled ("A" to "L" - Music and Movies categories; "A" to "O" Games and Sports categories), column by column, from top to bottom and from left to right. Based on the eye tracker data (duration and position of each fixation) the number and duration of the fixations inside each AOI were quantified.

Fig. 11 shows that in most categories, except for the "Sports" category, the icons belonging to the Object image-type representation received more fixations. In the "Sports" category the number of fixations for a few participants were very large, which biased the mean fixation count results. But if we look at the number of times (one for each participant) that each image-type representation obtained the higher mean fixation count, the Object image-type representation gets the best score (Fig. 12). The mean fixation duration results, presented in Fig. 13, also corroborate these findings. These results indicate that the icons belong- 


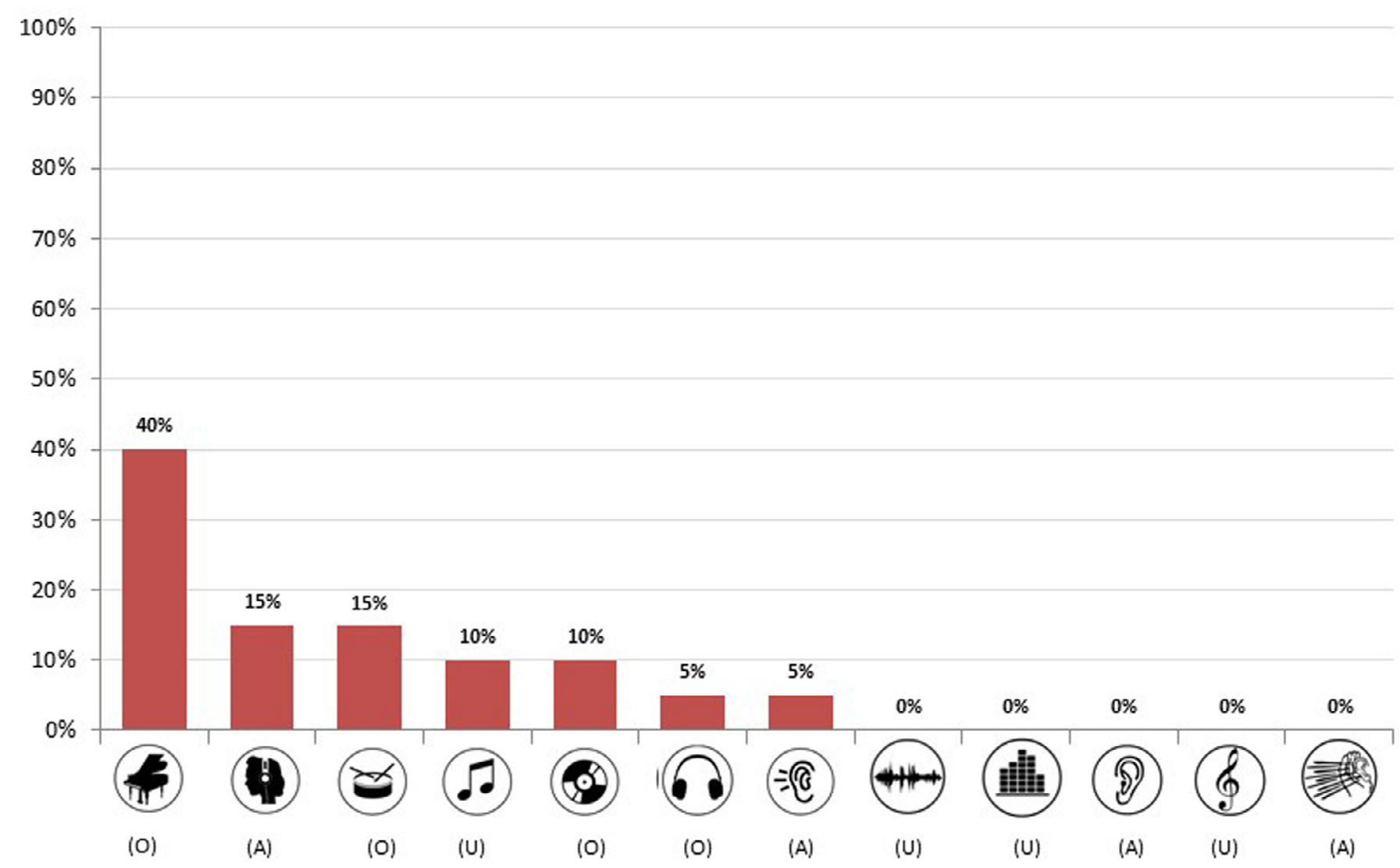

Fig. 6. Number of images selected for the "Music" category.

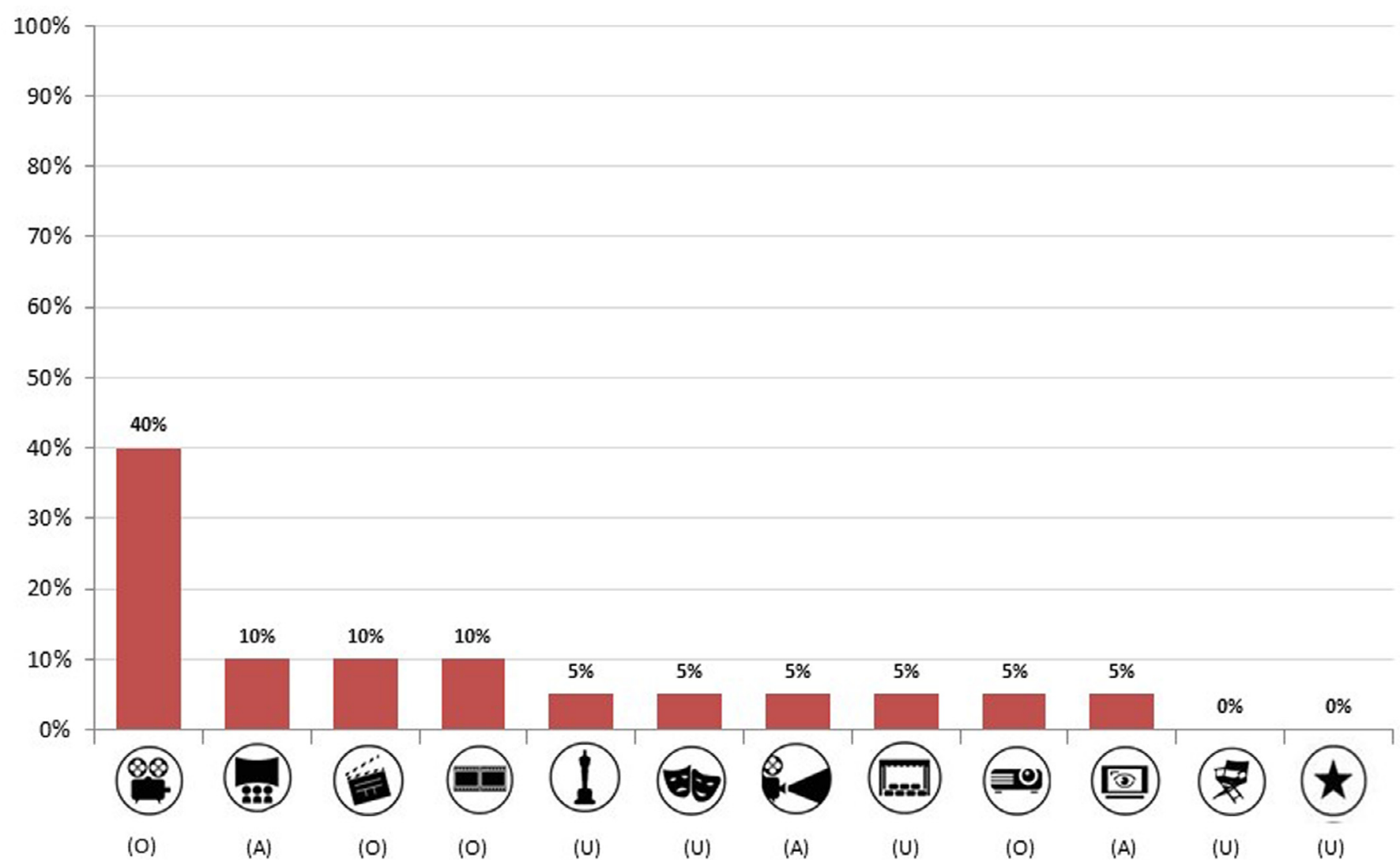

Fig. 7. Number of images selected for "Movies" category. 

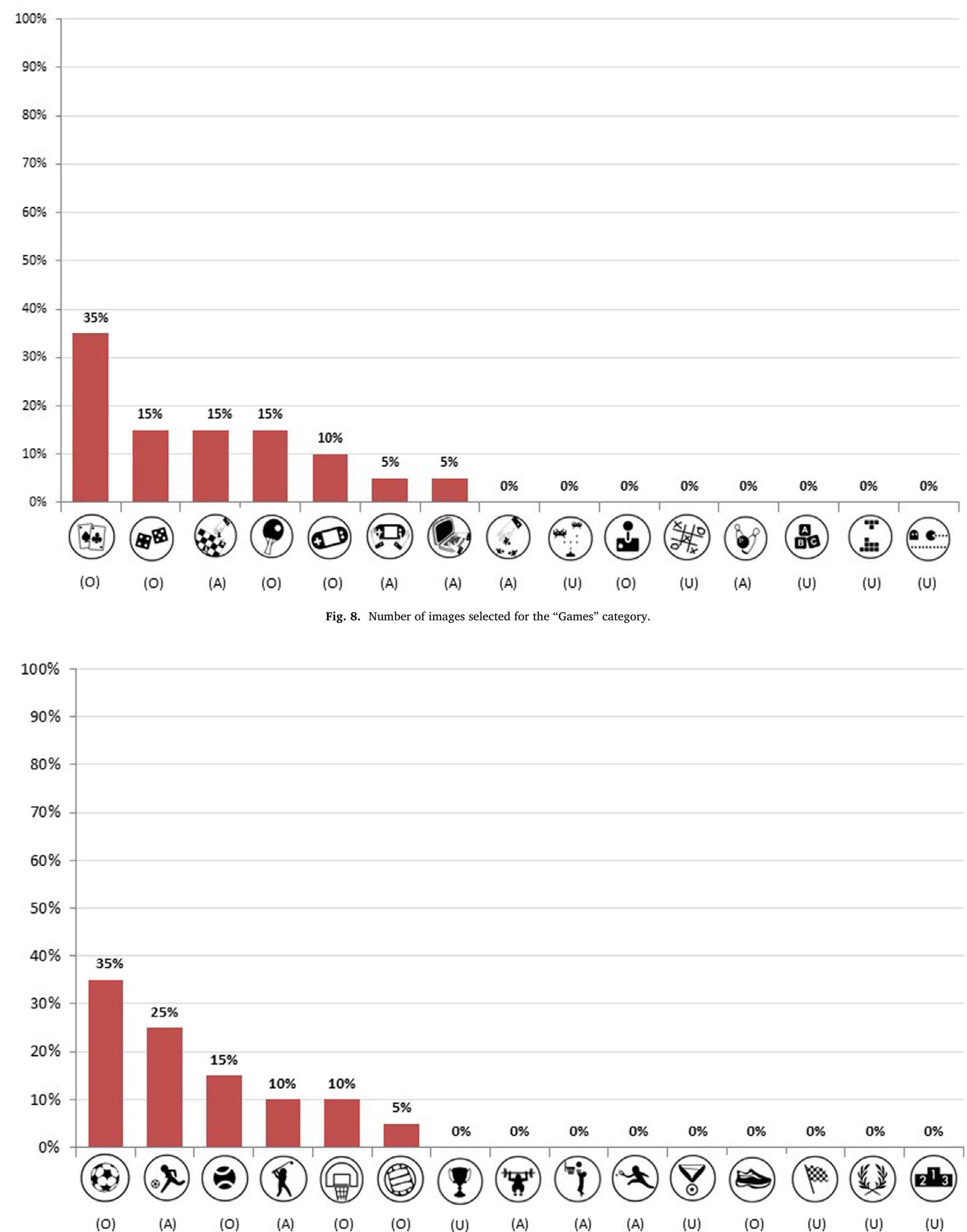

Fig. 9. Number of images selected in the "Sports" category. 

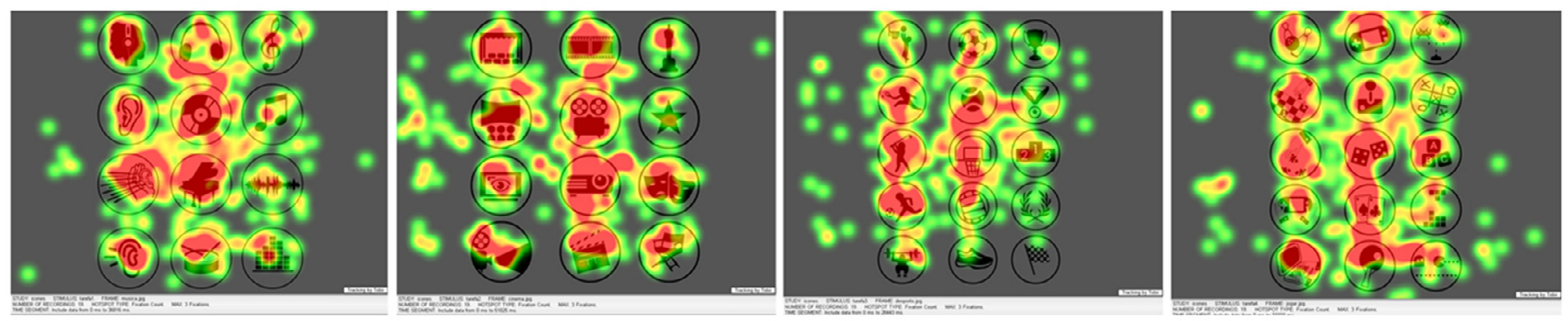

Fig. 10. Heat map results by category (music, movies, games, and sports).

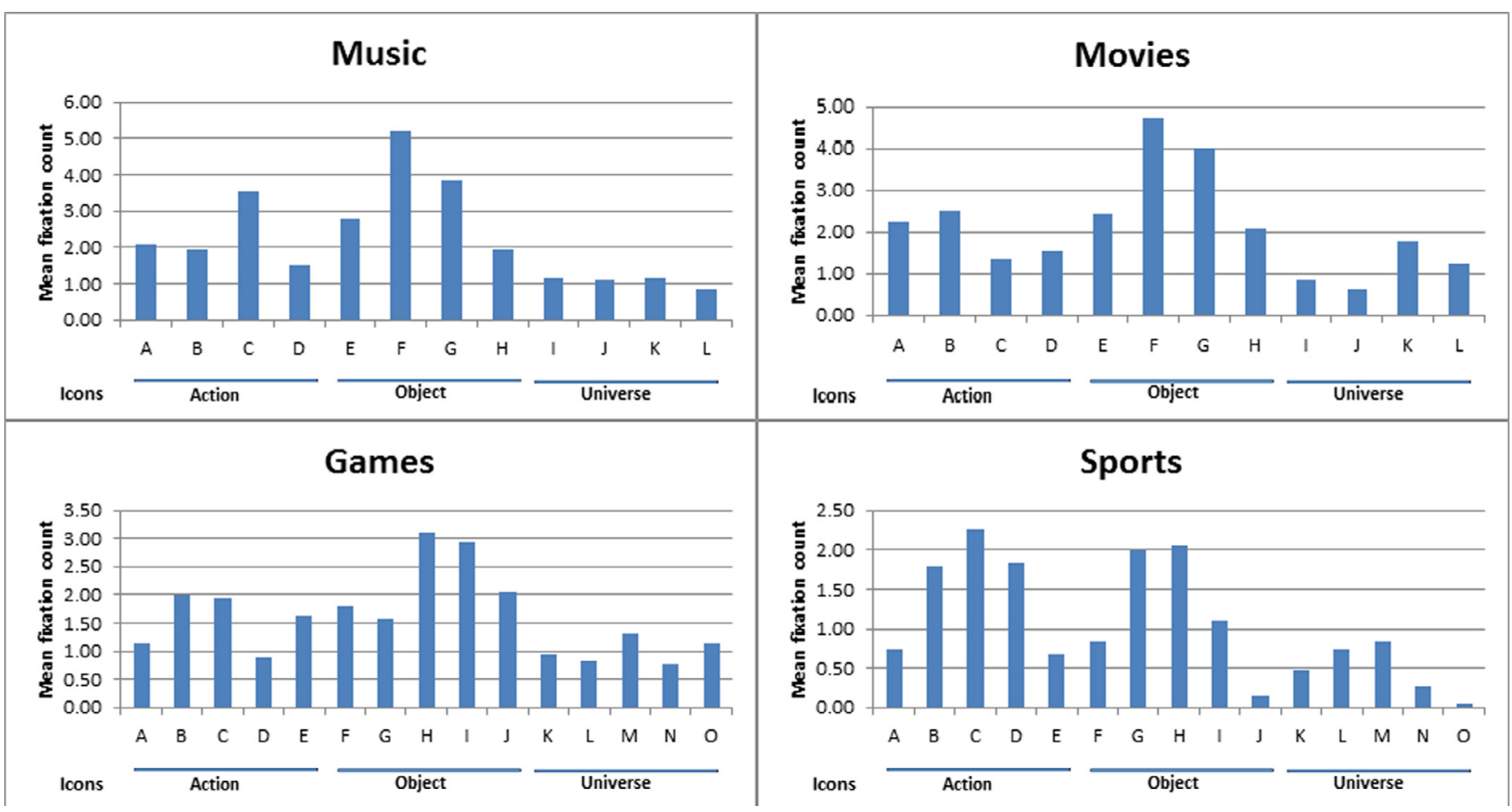

Fig. 11. Mean fixation count for each category.

Fixation count wins

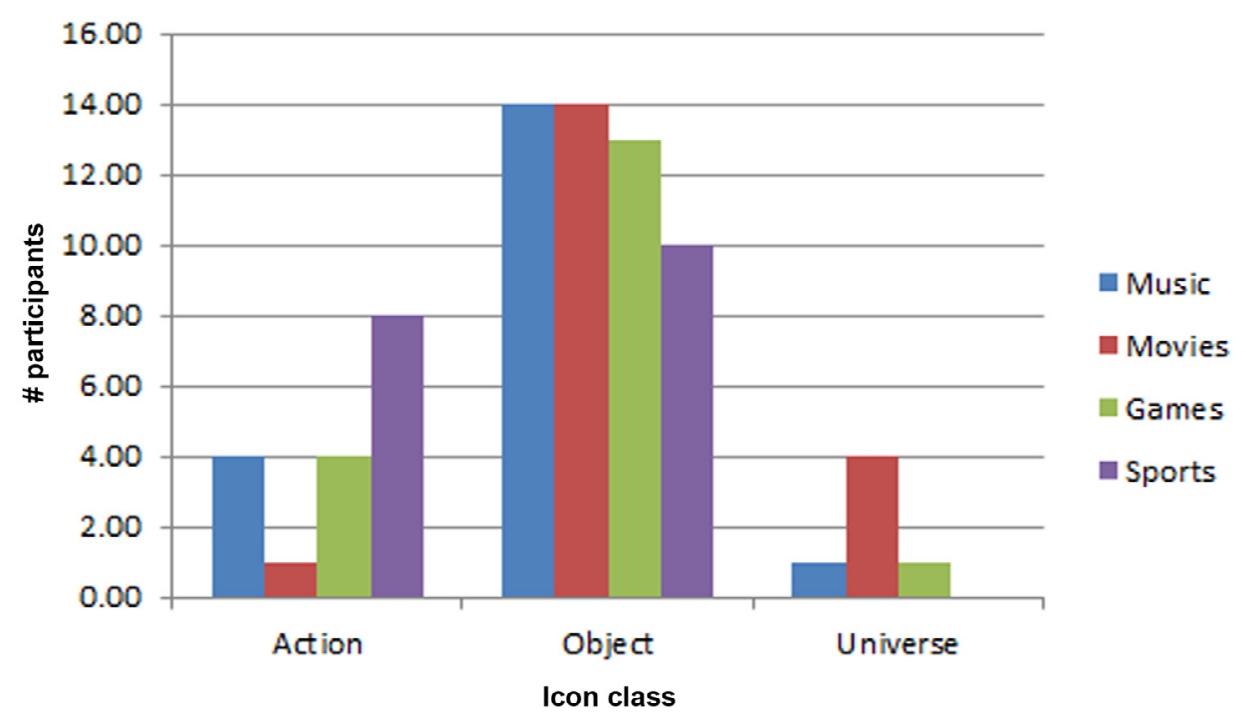

Fig. 12. Number of fixation count wins for each category. 


\section{Mean fixation duration}

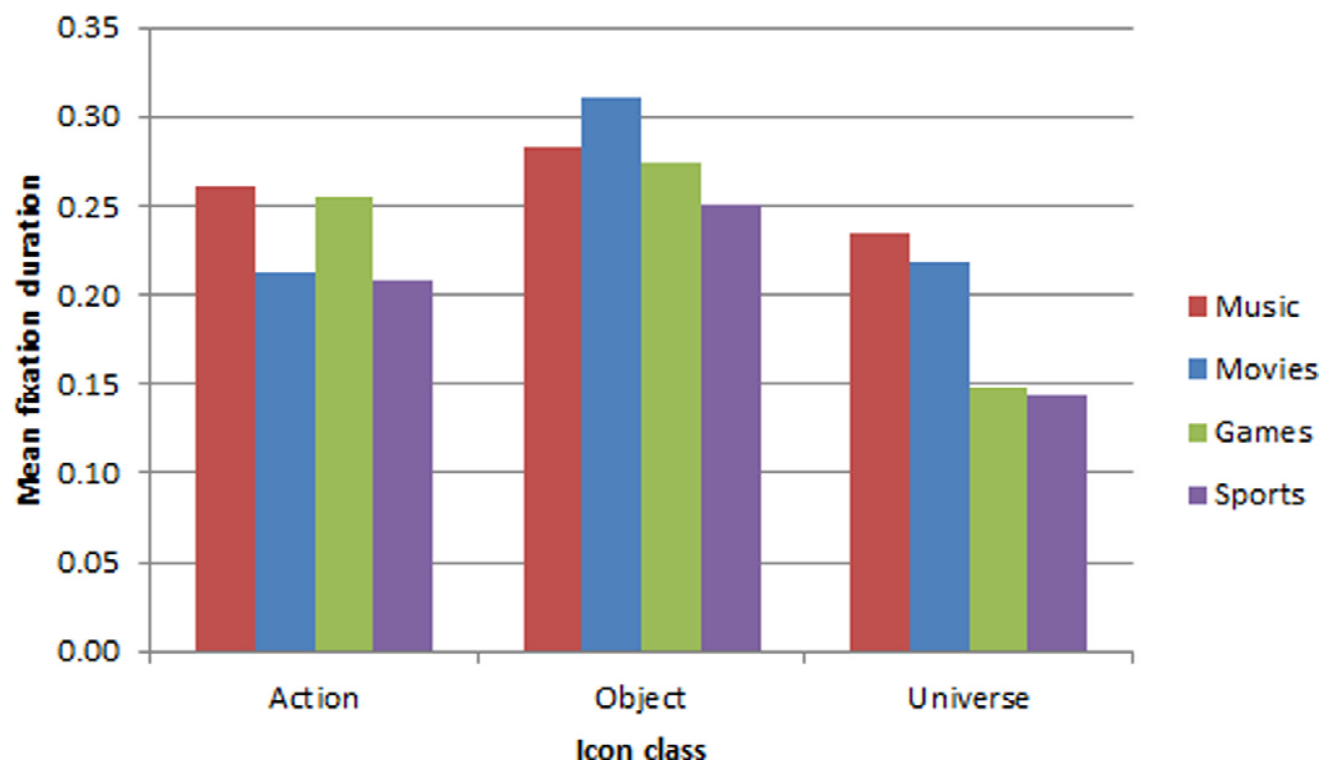

Fig. 13. Mean fixation duration for each category.

ing to the Object image-type representation attracted more attention, which supports our findings.

The icons chosen by the participants correspond most of the time (more than $83 \%$ ) to the icons with the highest or the second highest number of fixation counts or to a neighbour icon, indicating that usually the participants chose the image that they paid attention to.

\section{Discussion}

Globally, the results showed that the group preferred object representative images. The choices of these participants frequently related to known objects that they use and see daily. In other words, it appears that they preferred images that were simpler to decode and closer to the "signifier" - that is, images that were more concrete in their representation or represent specific objects (see Fig. 2). These results may suggest that some customisation of the images may be needed to make them coincide with everyday objects familiar to a specific group. These behaviours were also observed in the heat maps (Fig. 10) and in the eye tracker data analysis (Figs. 11-13), showing that the participants focused most of their attention on the Object and Action image-type, thus possibly indicating their preference for the Object or Action representative images as well as distance from the Universe image-type. Regarding the process of choosing the preferred images, it was observed that subjects usually chose the image that they paid more attention to.

Specifically, in the music category (Fig. 6), the participants preferred images that represent objects that they use on a daily basis. Universe representative images were almost never selected by the participants, possibly because of a lack of interest in this image-type (as suggested by the individual heat maps) or because the participants did not recognise the image representation. The only two participants that chose this type of representation did so because they had participated in music classes as an extra activity outside the school, e.g., their level of music literacy was higher and this probably changed their knowledge/context regarding this category. Another important aspect is that abstract images are commonly used to represent some details of our environment (Marganic, 2010), and also this type of images are very likely to be found (as an image hyperlink) in a digital environment, such as the treble clef and the music note. Moreover, different principles can be used for designing icons. The choice of significance for signs can be based on criteria such as, for instance, easy remembering, easy guessing, relation to population stereotypes. Given the many degrees of freedom underlying the design of iconic signs, a general principle should probably be adopted as a design aid in order to have an intuitive context for guiding users. However, we are increasingly distancing ourselves from the need for parallelism with the real world to understand the virtual context. A button does not need to graphically look like a real button so we can understand it. We are moving towards simplification and detachment from the real environment. A higher level of decoding is needed for abstraction (universe-type representation) content rather than the figurative (object-type representation). However, the designer should be aware that the content must be accessible and usable for all, regardless of their individual capabilities or characteristics.

The obtained results may indicate that these Universe image-type representations were not selected for the music category by more participants, because they did not associate those types of representations with the category.

In the movies category (Fig. 7), the choices of the participants resembled objects that the subjects saw either on television or during their visits to the cinema. When we asked them about their choices, they identified the video camera image as the movies category.

In the games category, the choices of the participants were objects that they recognised and used in their play activities, primarily in school (Fig. 8). Given that playing cards was the participants' most frequent game, in either a digital or physical environment, it was not surprising that the cards image was the one most selected for this category.

Also, in the sports category, the participants' choices coincided with object representative images (Fig. 9). Football has an important role in Portuguese sports education, and when we asked the participants about their choices, they highlighted this fact.

Furthermore, as mentioned, two of the categories, games and sport, had three more images (for a total of fifteen) than the other two categories (music and movies, a total of twelve images). The participants chose seven and ten images in the music and movies categories, respectively; in the games and sports categories, they chose seven and six images in total. One might expect that by having more options the number of different choices made by the participants would increase, but this has not occurred. This may indicate that it is the category that affects the dispersion of choices and not the number of icons. This result also 
indicates that probably there are categories where there is more concordance and it will be easier to select an appropriate image, and others where the number of options that needs to be considered is higher.

In short, in this study, we investigated the preferences of people with intellectual disabilities. The results suggest that for people with intellectual disabilities the best image-type representation is object representation.

The use of icons to represent categories, and thus navigate within a web page, is a common practice on the World Wide Web. Early studies have shown that people with intellectual disabilities perform better in navigating web sites when images are used as hyperlinks. The results obtained provide a basis for how we should direct our efforts to give people with intellectual disabilities the autonomy to perform some basic tasks on the internet. So, if they preferred object-type representative images to a given category, will they have more autonomy on a website that uses this image-type of representation for navigation purposes? Can this fact increase usability and accessibility of the websites?

Care should be taken with internal consistency, and thus it must be clear which icons represent navigational content and which are used only for aesthetics. More studies are needed to address these issues and to achieve the final goal of accessibility for people with intellectual disabilities.

\subsection{Limitations of the study}

In this paper, we investigated the preferences of people with intellectual disabilities regarding different image-type representation; we did not assess interpretability. Thus, more research is needed to understand whether the preferences of the subjects are affected by their abilities to decode the icons or by the aesthetics of the images.

Another limitation comes from the fact that some participants were involved in the image design process, as described in the materials section. Thus, participants could be biased to choose their drawings as that is what they are more familiar with.

Also, we did not randomise the icons within each category. Thus, we cannot state with a great deal of confidence that participants' choices are based on their personal preference and not because of their natural language reading direction. Further tests should be made to clarify this issue.

\section{Conclusions and future work}

The main goal of this study was to measure the user's preference for image-type representation (Object, Action and Universe), within four different categories (music, movies, sports, games), which can be used as hyperlinks in a digital navigation context. Image-type representation was designed according to three types of representation: Object (simpler to decode), Action, and Universe (more complex to decode).

The results showed that the participants preferred object-type representative images for all the categories tested (music, movies, sports and games). Their choice was influenced by activities and tasks that the participants performed and recognised on a daily basis at school or home, in digital or non-digital environments. Images that were not familiar in the participants' daily life, images more complex to decode and those with an abstract type of representation were mostly not selected.

These results indicate that image hyperlinks designed as object-type representation, because of its close connection to the representation, are simpler to decode (Fig. 2), and consequently could facilitate Web navigation of people with intellectual disabilities. We believe that if we design systems with hyperlinks that could be represented by images that are easily decoded by people with intellectual disabilities, it could help other groups of people that see their interaction diminishing, enhancing accessibility of the system.

Furthermore, semiotics and cultural issues associated with imagetype representation (such as colour, shape, and perspective) and society aspects (such as gender, age, geographical location, and socio-economic context) for better interpretability by people with intellectual disabilities is a topic that requires more research. In this paper, we did not intend to determine the best icon for each category, but instead to measure the user's preference for image-type representation (Object, Action, and Universe), within four categories.

In future work, we will study usability, where we will assess effectiveness and efficiency of hyperlinks for navigation using different image-type representations. Further tests will be conducted in which the icons' order will be randomized within each category to be sure that the natural language reading direction does not influence the participants' choices. Future studies will also consider whether preference and performance are correlated. Additionally, we intend to study preferences regarding image composition (photography, coloured cartoonish and black/white drawings) and image size.

\section{References}

Bohman, P.R., 2007. Functional Cognitive Disabilities. Retrieved from Access: June 29 2015. http://paulbohman.com/accessibility/cognitive/functional.

Bouquet, S., 2004. Introdução à Leitura De Saussure, Ninth ed. Translation of Carlos A. L. Salum e Ana Lúcia Franco, Cultrix, São Paulo.

Carey, C.C., Friedman, M.G., Bryen, D.N., 2005. Use of electronic technologies by people with intellectual disabilities. Mental Retardation 43 (5), 322-333.

Dix, A., Finlay, J., Abowd, G.D., Beale, R., 2004. Human-Computer Interaction, Third ed. Pearson- Prentice Hall, p. 101. 388-390; 388-390; 748. ISBN 0130-461091.

Endsley, M.R., 2016. Designing For Situation awareness: An approach to User-Centered Design. CRC Press.

Freeman, E., Clare, L., Savitch, N., Royan, L., Literhland, R., Lindsay, M., 2005. Improving website accessibility for people with early-stage dementia: A preliminary investigation. Aging Mental Health 9 (5), 442-448.

Freidman, M.G., Bryen, D.N., 2007. Web accessibility design recommendations for people with cognitive disabilities. Technol. Disabil. 19, 205-212 ISSN 1055-4181/07/\$17.00. IOS PRESS.

Harrysson, B., Svensk, A., Johansson, G., 2004. How People with Developmental Disabilities Navigate the Internet. British J. Special Educ. 31 (3), 138-142.

ISO 9241-21, 2010. Ergonomics of Human-System Interaction - Part 210: Human-centred design For Interactive Systems https://www.iso.org/standard/52075.html. Access: April, 10, 2017.

Marganic, M.Buzaši, 2010. Development visual literacy in digital environment. In: The 33rd International Convention MIPRO. Opatija, Croatia, pp. 1096-1098.

Nadin, M., 1988. Interface design: A semiotic Paradigm. Mouton de Gruyter, Amsterdam, pp. $269-270$.

Rocha, T., 2008. Acessibilidade e Usabilidade na Internet para Pessoas com Deficiência Intelectual MSc thesis. University of Trás-os-Montes e Alto Douro.

Rocha, T., Gonçalves, M., Godinho, F., Magalhães, L., Bessa, M., 2009. Accessibility and usability in the internet for people with intellectual disabilities. In: Proceedings of 2nd International Conference on Software Development for Enhancing Accessibility and Fighting Info-exclusion. Lisbon. DSAI'2009, pp. 25-29.

Rocha, T., Gonçalves, R.M., Magalhães, L.G., Bessa, M., 2012a. Web accessibility and digital businesses: the potential economic value of portuguese people with disability. In: Proceedings of 4th International Conference on Software Development for Enhancing Accessibility and Fighting Info-exclusion. Procedia Computer Science. DSAI 2012, Douro Region.

Rocha, T., Bessa, M., Gonçalves, M., Cabral, L., Godinho, F., Peres, E., Reis, M.C., Magalhães, L., Chalmers, A., 2012b. The recognition of web pages' hyperlinks by people with intellectual disabilities: an evaluation study. J. Appl. Res. Intellectual Disabil. 25 (6), 542-552. doi:10.1111/j.1468-3148.2012.00700.x.

Rocha, T., 2014. Metáfora de Interação para o Acesso à Informação Digital de uma Forma Autónoma por Pessoas com Deficiência Intelectual PHD thesis. University of Trás-osMontes and Alto Douro.

Rocha, T., Bessa, M., Magalhães, L., Cabral, L., 2015. Performing universal tasks on the Web: interaction with digital content by people with intellectual disabilities. Interacción'15 Proceedings of the XVI International Conference on Human Computer Interaction, Vilanova i la Geltrú, Spain September 07 - 09, 2015.

Roh, S.Z., 2004. Designing accessible Web-based instruction for all learners: Perspectives of students with disabilities and Web-based instructional personnel in higher education, Unpublished Doctoral dissertation. Indiana University. Retrieved from Access: November 22, 2015: https://mutex.gmu.edu:2068/pqdweb?did= $828407271 \&$ sid $=3 \& F m t=2 \&$ clientId $=31810 \&$ RQT $=309 \&$ VName $=$ PQD.

Saussure, F.de., 1996. Curso De Linguîstica Geral. Translation: Antônio Chelini et al., 25th ed. Cultrix, São Paulo.

Sevilla, J., Herrera, G., Martinez, B., Alcantud, F., 2007. Web accessibility for individuals with cognitive deficits: a comparative study between an existing commercial web and its cognitively accessible equivalent. ACM Trans. Comput.-Human Interaction 14 (3) article 12 .

Small, J., Schallau, P., Brown, K., Ettinger, D., Blanchard, S., Krahn, G., 2005. Web Accessibility for People with Cognitive Disabilities Retrieved from Access: November 21, 2015. http://www.resna.org/ProfResources/ Publications/Proceedings/2005/Research/CAC/Small.php. 
Uden \& Dix, 2000. Iconic Interface for Kids on the Internet Unpublished work. Retrieved from: Access: December 12, 2015. And http://alandix.com/academic/papers/ kids-icons-2000/kids-icons-2000.pdf. Accessed: August 8, 2016. usability. gov - Improving the User experience https://www.usability.gov/what-and-why/ user-experience.html. Access: April, 10, 2017. http://citeseerx.ist.psu.edu/viewdoc/ summary?doi=10.1.1.22.7205.

Wiebe, M., Geiskkovitch, D., Bunt, A., Young, J., Glenwright, M., 2016. Icons for kids: can young children understand graphical representations of app store categories? In: Proceedings of Graphics Interface 2016. Victoria, British Columbia, Canada, 1-3 June 2016, pp. 163-166. doi:10.20380/GI2016.20.
Yates, D., Moore, D., McCabe, G., 1999. The Practice of Statistics, 1st Ed. W.H. Freeman, New York.

Zarin, R, 2009. Mejla Pictogram 2.0. Institute of Design in Umea, Sweden and Swedish Institute for Special Needs Education Retrieved from: Access: February 52013. http://216.46.8.72/tmp/v2/images/pictoCom/Final_report_Pictogram2.pdf. 